\title{
Child computer interaction: advances in methodological research
}

\author{
Introduction to the special issue of cognition technology and work
}

\author{
Panos Markopoulos · Janet Read \\ Johanna Hoÿsniemi · Stuart MacFarlane
}

Published online: 27 March 2007

(C) Springer-Verlag London Limited 2007

In recent years, there has been an increasing trend for children to use information and communication technology in its various forms. Children now grow up immersed in technology to a level that keeps surprising earlier generations, but which, to them, is simply an inherent element of their habitat. Although this immersion is partly dependent on wealth and circumstance, it is certainly the case that in most developed countries children are frequently users and owners of Personal Computers, video game consoles, personal music technologies and mobile phones.

This increase in usage of interactive technology by children has not gone unnoticed. More than ever before, technology manufacturers and service providers are turning their attention to children as a growing market segment. Even more important, societies are becoming concerned to ensure that appropriate products and services, namely those that can support development and enhance well-being, are being made available for children. Whatever motivates the design of interactive technology for children, it is clear that there is an urgent and present need for methodological knowledge about the design of these products and an

\footnotetext{
P. Markopoulos ( $\square)$

Department of Industrial Design,

Eindhoven University of Technology,

Eindhoven, The Netherlands

e-mail: p.markopoulos@tue.nl

J. Read · S. MacFarlane

Department of Computing,

University of Central Lancashire, Preston, UK

J. Hoÿsniemi

Department of Computer Science,

University of Tampere, Tampere, Finland
}

understanding of the ways in which interaction takes place between the child and the technology.

Designing technology for humans has been studied for many years. Initially concentrating on ergonomics of use, before becoming more concerned with general human factors, this field has now matured to the point where there are defined research areas that have clear identities. Human computer interaction (HCI) is that area that focuses on the interaction between man and machine. HCI has been growing in importance over the last 25 or more years, and, as a discipline, has matured and settled. For HCI practitioners and academics there are published curricula, dedicated high-impact journals, specialised undergraduate and postgraduate University courses, and vibrant associations of professionals in the field (e.g., Usability Professionals Association, British HCI group, ACM SIGCHI).

Child computer interaction (CCI) is the sub-field of HCI that studies how children use interactive products. In contrast with $\mathrm{HCI}, \mathrm{CCI}$ is still finding its way. Relating to sociology, education and educational technology, connected to art and design, and with links to storytelling and literature, as well as psychology and computing this new field borrows methods of inquiry from many different disciplines. This disparity in methods of enquiry makes it difficult for researchers to gain an overview of research, to compare across studies and to gain a clear view of cumulative progress in the field.

It is difficult to identify an exact moment when CCI became a specialised field as it was a gradual maturity of the area that spawned its creation. In the early days, pioneering work by Papert and Resnick at the Massachusetts Institute of Technology (MIT) laid the foundations for work that was carried forward by a few interested individuals around the globe. Several key individuals including 
Rogers, Scaife, Druin, and Kafai accumulated experience in the design of technologies for children, and in so doing, developed new methodologies for design and accumulated experience in the form of case studies. A representative record of these works can be found in the seminal volume edited by Druin (1998).

In recent years, the field of CCI has witnessed steady growth. In 2002, the increasing number of CCI related publications appearing at a range of general interaction conferences triggered the establishment of a specialized annual conference series entitled "Interaction Design and Children", and recent years have seen workshops and events at several major HCI conferences where interaction and children has been the specific focus. As the research community has grown, there is a need to collect together results and advances in methodologies for designing children's products, including newly developed methods and studies documenting experiences of existing methods.

The papers in this special issue reflect the growing maturity of the CCI field. Not only are novel methods presented and rationalized, but also advances in methodology transcend simple experience reports, by seeking experimental validation of the validity of the methods. Experiments are reported that profile the relative performance of methods against competitor methods and authors of the collected works explicitly seek to inform the development of such methods upon theoretical works including, for example, Norman's theory of action (Norman 1986), Activity Theory (Nardi 1996) and Malone and Lepper's (1987) investigation of what makes games fun.

The applicability and usefulness of participatory design methods have generated lot of interest in the field of childcomputer interaction; however, socio-cultural activity theory has only recently been considered as a practice within the field. Iversen and Brodersen have taken this framework into use and introduce the BRIDGE method which contains a palette of design techniques where children are involved in the design on the same terms as adults. The method has been developed over a 5-year period within two consecutive research projects. The paper first discusses in depth the methodological issues of socio-cultural approaches in CCI and the BRIDGE method, and continues with three examples that illustrate how the method can be applied in different design contexts.

Barendregt, Bekker and Baauw describe the development and testing of the Problem Identification Picture Cards (PIPC) method. This is a method for evaluating games that helps young children aged 4-5 to express opinions about fun and usability issues by selecting relevant picture cards. Young children do not always have the confidence or the vocabulary to express their opinions regarding the game evaluated; this method can be used as a replacement for, or a supplement to, an interview or observation method. The paper discusses the design of the cards used and goes on to describe a comparative study between PIPC used with think-aloud, and think-aloud alone. The data confirms the authors' hypotheses concluding that more issues regarding the interaction design were raised by using the combined method, the number of verbalisations by children was not reduced, and children liked the combined method at least as much as think-aloud on its own.

Zaman demonstrates contextual laddering, a method that can be used to research the likeability of products for children. With its focus on this facet of usability that is seldom found in adult studies, the Zaman paper demonstrates the need to consider not only new methods for old problems but also the requirement, in this new field, to ensure coverage of new problems. The paper provides an exploratory analysis of the method which includes the results from two instances of use.

Read discusses the problems of surveying children's opinions as part of a product evaluation. The paper presents the newest iteration of the Fun Toolkit, a survey instrument for children that was originally proposed 5 years ago. This toolkit, that focuses on the fun aspects of a product has matured alongside the research field and this reflective paper examines its use and its usefulness, drawing on the experiences of several researchers that have used the toolkit. Read discusses these experiences and presents a quantitative analysis to support the validity of the instrument developed. The paper describes a new version of the Fun Toolkit and outlines how it should be used with children.

Bekker, Baauw and Barendregt present another validation study in which they compare their own method for evaluating computer games, the Structured Expert Evaluation Method (SEEM) against Heuristic Evaluation, used with an extended set of heuristics to cover both usability and fun. SEEM is an inspection method, extending earlier usability inspection methods with questions that aim to assess the fun children have with a product. The authors not only demonstrate that SEEM outperforms heuristic evaluation in this context, but in doing so also provide an excellent example of how to carry out such experimental method comparisons. A range of issues arise regarding the validity of such comparisons and advance the current state of the art in comparative studies of evaluation methods.

The paper by Roussou, Oliver and Slater describes the exploratory use of Activity Theory as a tool for the analysis of behaviour of children interacting with an immersive Virtual Environment. The overall aim of the research project, of which the study reported is a part, was to study the connections between interactivity, learning and conceptual change. This is an interesting and novel use of Activity Theory to analyse qualitative data. A small num- 
ber of children were observed and recorded, and the paper describes in detail how Activity Theory was used to provide the designers with an understanding of the children's interaction behaviour during their attempts to build virtual columns up from component parts.

User representation methods, especially personas, have become popular both in interface design and in usability testing. However, child-specific methods for creating personas have not been fully explored in the field of childcomputer interaction. For the first time Antle provides child-persona framework that is based on rich theoretical knowledge of children. The proposed framework contains three dimensions which are childhood needs, developmental abilities and experiential goals. The paper discusses these dimensions in-depth and provides concrete examples how to apply this framework in real-life design contexts with children. The method allows designers to create child abstractions when participatory design practices are limited or impossible due to policy, legal or ethical reasons.

The collection of papers presented in this special issue is not intended to provide a representative coverage of the field of Child computer interaction but it does offer the reader an opportunity to appreciate the possibilities and the progress in the research area. It captures some of the main advances at this moment in methodological research and as a literature resource it can open doors for readers into this exciting area of research.

As would be expected in such a young area, the authors of the papers are eager to highlight the fact that there are still many open questions and unresolved issues to be grappled with. For example, the authors of the SEEM paper highlight a need for further work to determine its fit within a design process and its possibilities for diverse types of interactive products. In her reflection upon the Fun Toolkit, Read recognises that there is still work to be done to discover which constructs need to be measured and how these constructs map to engagement and software appeal. A whole host of questions emerge from the papers in this volume which could be tackled by future research: what is the range of interactions for which Activity Theory provides a useful analysis? Are such theory based analyses an efficient means for finding uncovering usability problems? Who are the children best suited to the PIPC method rather than other more estab- lished methods, and in what ways can the method be extended or applied in different situations?

The future for CCI is tantalising. Technologies as yet unimagined will be the playthings of future generations and experiences unlike any experienced to date will fill children's hours. The current research generation can no more predict the impacts of these advances than our forefathers could have predicted the impacts of the MP3 player but effort invested to discover methods to evaluate and design children's products and services will be time well spent.

Future technologies will still be placed in the context of children's lives, and so methodological research needs to extend to the possibilities of evaluating technology use as part of children's daily activities at home, school or at play, to support the evaluation of long term use of products in such contexts and to eventually reflect upon the impacts of these products upon children's lives. This challenge will require stronger links to the domains of educational technology and an extension of methods of inquiry to include field trials and field work. Such developments will result in this growing subfield achieving a significant impact on industry and, by implication, on children's lives. Equally important is ensuring the practical relevance of the methods proposed.

In assembling this collection of papers, the editors' hope that readers will examine the works described and will take on some of the solutions and the research challenges proposed within them. In this way, the children of today and of the future might have technologies that fit their needs, will be better designed, better motivated and better fit for purpose.

\section{References}

Druin A (1998) The design of children's technology. Morgan Kaufmann, San Francisco

Malone TW, Lepper MR (1987) Making learning fun: a taxonomy of intrinsic motivations for learning. In: Snow RE, Farr MJ (eds) Aptitude, learning and interaction iii cognitive and affective process analysis. Erlbaum, Hillsdale

Nardi B (1996) Context and consciousness: activity theory and human-computer interaction. MIT, Cambridge

Norman DA, Draper SW (1986) User centered system design: new perspectives on human-computer interaction. Erlbaum, Hillsdale 